\title{
Wavelength-Insensitive Nonadiabatic Mode Evolution Couplers
}

\author{
George T. Paloczi, Student Member, IEEE, Avishay Eyal, Member, IEEE, and Amnon Yariv, Life Fellow, IEEE
}

\begin{abstract}
We investigate the wavelength insensitivity and fabrication error tolerance of the relative output amplitude and phase for a new type of optical coupler. This new coupler overcomes the long-length requirements of adiabatic couplers, yet achieves similar desirable properties. The three-space geometrical representation of coupled-mode theory, used as the design and analysis method, is presented and an example of a 3-dB coupler is examined.
\end{abstract}

Index Terms-Coupled-mode theory (CMT), integrated optics, optical couplers, optical waveguides, Poincaré sphere.

\section{INTRODUCTION}

$\mathbf{T}$ WO-BY-TWO optical couplers that maintain output differential phase and relative amplitude conditions despite operating wavelength variation and fabrication errors are of utmost importance in integrated optics, especially for cascaded filters and modulators [1]. To this end, Mach-Zehnder structures can be constructed with a good degree of tolerance in the output amplitudes [2]; however, in such constructions, the output differential phase changes maximally with wavelength variations, rendering these devices less useful in filter applications. The most promising technology for achieving the desired tolerances has been the adiabatic coupler [3], for which the local system states follow adiabatically the local eigenstates of the waveguiding system and, thus, mode conversion between eigenstate populations is minimized. However, this requires structures that change over distances much longer than the beat length at any cross section of the coupler, thus limiting the usefulness of adiabatic couplers. Here, we describe a novel design approach, which is based on varying the local eigenstates, while keeping their beat length constant. It is shown that, by choosing the beat length with discretion while relaxing adiabatic criteria, adiabatic-like wavelength variation immunity and fabrication tolerance can be realized for much shorter device lengths.

Manuscript received August 26, 2003; revised October 14, 2003. This work was supported in part by the Office of Naval Research, by the Defense Advanced Research Project Agency, and by the Air Force Office of Scientific Research.

G. T. Paloczi and A. Yariv are with the Department of Applied Physics, California Institute of Technology, Pasadena, CA 91125 USA.

A. Eyal was with the Department of Applied Physics, California Institute of Technology, Pasadena, CA 91125 USA. He is now with the Department of Interdisciplinary Studies, Tel Aviv University, Tel Aviv 69978, Israel.

Digital Object Identifier 10.1109/LPT.2003.821254

\section{THEORETICAL BACKGROUND}

Coupled-mode theory (CMT) establishes that the vector of complex field amplitudes $\overrightarrow{\mathbf{E}}=\left[E_{1} E_{2}\right]^{T}$ for two weakly coupled lossless waveguides evolves according to

$$
\frac{d \overrightarrow{\mathbf{E}}}{d z}=\tilde{\mathbf{C}} \overrightarrow{\mathbf{E}}
$$

where $\tilde{\mathbf{C}}(\mathbf{z})$ represents the coupling matrix with local propagation constants of the individual waveguides $c_{11}=-i \beta_{1}$ and $c_{22}=-i \beta_{2}$ as diagonal elements and the coupling coefficients between the waveguides $c_{21}=c_{12}=-i \kappa$ on the off diagonals [4]. Here, we assume the convention that $\kappa$ is real. In converting to the geometrical representation of CMT [5]-[7], the normalized amplitude vector at some position $z_{0}, \hat{\varepsilon} \equiv \vec{E} / \sqrt{\overrightarrow{E^{\dagger}} \vec{E}}$, becomes a unit state vector in a real three-dimensional space using the relation

$$
s_{k}=\hat{\varepsilon}^{\dagger} \sigma_{k} \hat{\varepsilon}
$$

where $\sigma_{k}$ are the Pauli spin matrices in the order

$$
\sigma_{1}=\left(\begin{array}{cc}
1 & 0 \\
0 & -1
\end{array}\right) \quad \sigma_{2}=\left(\begin{array}{cc}
0 & 1 \\
1 & 0
\end{array}\right) \quad \sigma_{3}=\left(\begin{array}{cc}
0 & -i \\
i & 0
\end{array}\right) .
$$

The coupling matrix $\tilde{\mathbf{C}}(\mathbf{z})$ maps to the same real three-dimensional space as a rotation vector

$$
\Omega_{k}=-i \operatorname{Tr}\left(\tilde{\mathbf{C}} \sigma_{k}\right) .
$$

Using (2) and (4) in conjunction with (1), the transformed coupled-mode equations are [6], [8]

$$
\frac{d \mathbf{s}}{d z}=\mathbf{\Omega} \times \mathbf{s} .
$$

The coupled-mode equations are now regarded dynamically as sequential infinitesimal rotations of the instantaneous state vector $\mathbf{s}\left(z_{0}\right)$ on the unit sphere around the instantaneous rotation axis $\boldsymbol{\Omega}\left(z_{0}\right)$. As seen from (5), a state vector is stationary when collinear with the rotation vector, thus, the antipodal points $\pm \hat{\boldsymbol{\Omega}}$ represent the two local eigenstates of the waveguide system. For nonconstant $\boldsymbol{\Omega}(z)$, a solution of (5) is obtained by direct numerical integration. This pictorial representation of a two-state system is analogous to the Poincare sphere of polarization optics [8].

\section{COUPLER Design}

For the coupling matrix above, the rotation vector becomes $\boldsymbol{\Omega}=\left[\begin{array}{lll}2 \Delta \beta & -2 \kappa & 0\end{array}\right]^{T}$, where $2 \Delta \beta \equiv\left(\beta_{2}-\beta_{1}\right)$. Accordingly, we consider only rotation vectors $\Omega$ that lie on the equator of the sphere, with $|\boldsymbol{\Omega}|=2 \sqrt{\operatorname{Im}(\kappa)^{2}+\Delta \beta^{2}}$. Factorization of the rotation vector into $\boldsymbol{\Omega}=|\boldsymbol{\Omega}(z)|[\cos (\theta(z)) \sin (\theta(z)) 0]^{T}$ gives as 


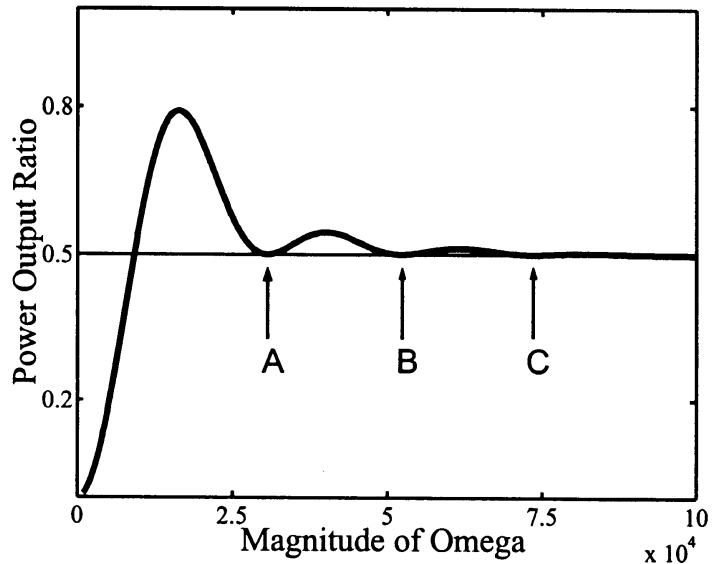

Fig. 1. Power output ratio as a function of $|\Omega|$ for the raised cosine shape function. Points A-C represent couplers with the desired 3-dB power output ratio.

the two design degrees of freedom the magnitude of the rotation vector $|\boldsymbol{\Omega}(z)|$ and the shape function $\theta(z)$. It should be noted that while the rotation vector is confined to the basal plane, the state vector might take any position on the sphere.

We now describe via an example how the above formalism can be used for the design of novel couplers with unique features. The device we examine is a $3-\mathrm{dB}$ nonadiabatic mode evolution coupler that, unlike a conventional directional coupler, yields no phase shift between the output waveguides. In choosing the shape function $\theta(z)$ to obtain the desired $3-\mathrm{dB}$ power output ratio and phase relationship, we require the initial and final eigenstates, assuming light is input in waveguide 1 , to be $\hat{\boldsymbol{\varepsilon}}^{\text {initial }}=\left[\begin{array}{ll}1 & 0\end{array}\right]^{T}$ and $\hat{\boldsymbol{\varepsilon}}^{\mathrm{final}}=(1 / \sqrt{2})\left[\begin{array}{ll}1 & 1\end{array}\right]^{T}$, corresponding in the geometrical representation to $\hat{\Omega}^{\text {initial }}=\left[\begin{array}{lll}1 & 0 & 0\end{array}\right]^{T}$ and $\hat{\mathbf{\Omega}}^{\text {final }}=\left[\begin{array}{lll}0 & 1 & 0\end{array}\right]^{T}$. We thus need a smoothly changing function with beginning and ending points $\theta=0$ and $\theta=\pi / 2$. Various shape functions have been compared [9], however, here, we choose for illustrative purposes, the raised cosine function

$$
\theta(z)=\left(\frac{\theta_{0}}{2}-\frac{\pi}{4}\right)\left(\cos \frac{\pi z}{l}-1\right)+\theta_{0}
$$

where $l$ is the half-length of the coupler and $\theta_{0}$ is an additive constant preventing $|\kappa|=0$ (infinite waveguide separation). The magnitude of the rotation vector is taken to be constant throughout the length of the 3-dB portion of the coupler for simplicity. Beyond the point where the desired 3-dB output is obtained, the magnitude of the rotation vector can be exponentially decreased, linearly separating the waveguides. This latter portion is of little consequence in the current study and is ignored in what follows. As discussed previously [10], to obtain the desired power output ratio, it is important to carefully choose specific ranges of the product $|\boldsymbol{\Omega}| \cdot l$ for a given shape function. As optical component miniaturization and integration progresses and as planar lightwave circuit complexity grows, coupler lengths are severely restricted so it is more realistic in this point of view to consider the length as restricted to a constant, and to carefully select ranges of $|\boldsymbol{\Omega}|$ to obtain the desired output. Fig. 1 shows a plot of the power output ratio for the raised cosine shape function as a function of $|\Omega|$. As can be seen, there exist several
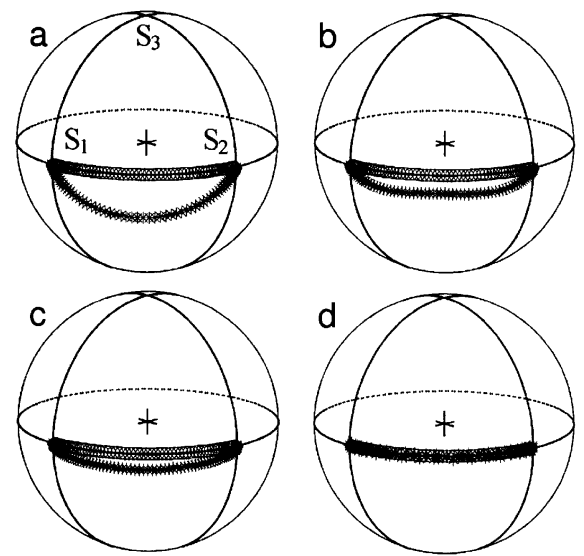

Fig. 2. Geometrical representation of the eigenstate (o) and system state $(x)$ trajectories. The system states begin occupying one eigenmode, i.e., light is injected into waveguide 1, and end in the eigenmode given by equal output power and phase. (a)-(c) depicts trajectories as calculated for $|\Omega|$ given by points A-C, respectively, for Fig. 1. In (d), we show the coincident system state and eigenstate trajectories of a long-length adiabatic coupler for comparison.

values of $|\boldsymbol{\Omega}|$ where the desired power output ratio (0.5) is obtained. Shape functions exhibiting smaller ripple exist, but at the cost of larger $|\boldsymbol{\Omega}|$ or longer length. Larger $|\boldsymbol{\Omega}|$ results in couplers that change rapidly in shape, increasing scattering loss, and alternatively longer lengths are against the spirit of this study. Referring to Fig. 1, the perfectly adiabatic limit results from $|\Omega| \cdot l \rightarrow \infty$.

Once the magnitude $|\boldsymbol{\Omega}(z)|$ and shape function $\theta(z)$ are chosen to achieve the desired output ratio and phase, the refractive index profile is computed as follows. For simplicity, we model the system as a slab waveguide structure of length $300 \mu \mathrm{m}$ with core and cladding refractive indexes of 1.55 and 1.5. The first element of the rotation vector immediately yields the individual propagation constants $\beta_{1}(z)=\bar{\beta}+\Delta \beta(z)$ and $\beta_{2}(z)=\bar{\beta}-\Delta \beta(z)$, assuming a constant average propagation constant $\bar{\beta}=\left(\beta_{1}+\beta_{2}\right) / 2$ that ensures single-mode operation. From these, the waveguide widths are obtained by inverting the solutions for the propagation constants of slab waveguides, as detailed in [4]. Finally, the second element of the rotation vector is used in the equation relating the coupling coefficients to the waveguide widths and center-to-center separation.

\section{WAVELENGTH INSENSITIVITY AND FABRICATION TOLERANCE}

Consider points $\mathrm{A}-\mathrm{C}$ in Fig. 1, where the magnitude of the rotation vector yields couplers exhibiting the desired 3-dB power splitting and phase relationship. To give a geometrical understanding to couplers designed with $|\Omega|$ at these points, we calculate the state vector trajectories and plot them in the geometrical representation along with the eigenstate trajectories in Fig. 2(a)-(c), corresponding with the letter designations in Fig. 1. These trajectories represent the crossing of the final state and the final desired state. With increasing $|\boldsymbol{\Omega}|$, the trajectories tend toward adiabatic behavior, for which the state vector and eigenstate remain arbitrarily close, as in Fig. 2(d).

To illustrate the wavelength insensitivity of the power output ratios and relative output phases for such nonadiabatic $3-\mathrm{dB}$ coupling structures, we calculate the state vector trajectories as 

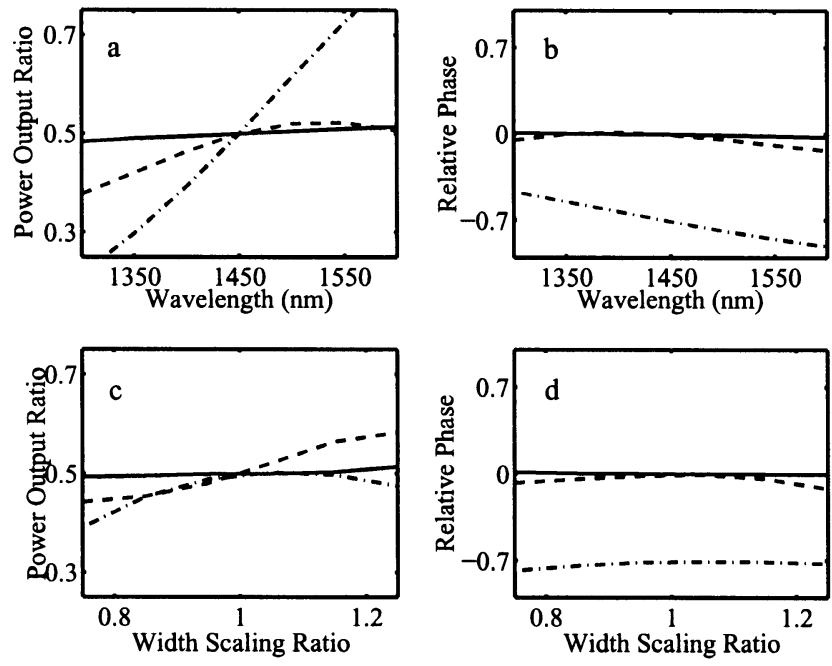

Fig. 3. (a) and (b) Power output ratios and relative output phases as a function of wavelength and (c) and (d) width scaling factor. The dashed-dotted (- - -) line represents a symmetric directional coupler for comparison. The dashed (- - ) and solid (-) lines give the output ratios and relative phases corresponding to couplers designed with $|\Omega|$ as given by points A and B in Fig. 1, respectively.

a function of wavelength using the model parameters described above. The power output ratios are plotted in Fig. 3(a), over a wavelength range of 1300-1600 $\mathrm{nm}$. For comparison, the wavelength dependence of a standard symmetric directional coupler is also shown. Clearly the coupler designed using $|\Omega(z)|$ chosen at point $\mathrm{A}$ is a marginal improvement over a directional coupler, but the choice of $|\boldsymbol{\Omega}(z)|$ at point B yields a coupler with a good degree of wavelength invariance. Point $\mathrm{C}$ gives even further improvement, but is not shown, as it essentially matches the desired $3-\mathrm{dB}$ output throughout the band. The relative output phases are shown in Fig. 3(b) and, as for the case of the power output ratio, a good degree of phase invariance is obtained for the choice of $|\boldsymbol{\Omega}(z)|$ at point B in Fig. 1 .

In photo- or electron-beam lithography used for the production of optical integrated circuits, an exposure error may result in a disparity of the intended waveguide widths and actual values. To simulate this, we calculate the power output ratio and output phases while varying the intended waveguide width via a scaling factor ranging from 0.75 to 1.25 . The power output ratio results as calculated using matrix methods are shown in Fig. 3(c) and the corresponding relative output phase calculations are shown in Fig. 3(d). Again, as in the case of wavelength variations, we see marked improvement over a directional coupler when $|\boldsymbol{\Omega}(z)|$ is chosen as point B of Fig. 1.

As an independent corroboration of the results as calculated using the matrix methods, beam propagation method (BPM) simulations [11] were employed. Results of the simulations agree quite well with the wavelength and scaling factor responses, as predicted by the matrix method.

\section{CONCLUSION}

We have presented a scheme for the design of optical couplers with a high degree of wavelength insensitivity and fabrication tolerance. It has been demonstrated that it is possible to obtain properties similar to those of purely adiabatic couplers, i.e., good wavelength insensitivity and fabrication tolerances, if the design parameters are chosen with care. The physical reason for the adiabatic-like features is due to the fact that, as the wavelength is varied or the waveguide shapes are scaled, $|\boldsymbol{\Omega}(z)|$ is changed by some amount. The key feature, therefore, to attaining the desirable properties of adiabatic couplers, is the slope of the curve shown in Fig. 1 near the points of 3-dB output. The smaller the slope, the smaller the deviation from the desired output.

\section{ACKNOWLEDGMENT}

The authors gratefully acknowledge the helpful discussions with J. Choi, W. Green, Dr. B. Marshall, Prof. S. Mookherjea, and Dr. J. Scheuer.

\section{REFERENCES}

[1] C. K. Madsen and J. H. Zhao, Optical Filter Design and Analysis. New York: Wiley, 1999.

[2] B. E. Little and T. Murphy, "Design rules for maximally flat wavelength-insensitive optical power dividers using Mach-Zehnder structures," IEEE Photon. Technol. Lett., vol. 9, pp. 1607-1609, Dec. 1997.

[3] Y. Shani, C. H. Henry, R. C. Kistler, R. F. Kazarinov, and K. J. Orlowsky, "Integrated optic adiabatic devices on silicon," IEEE J. Quantum Electron., vol. 27, pp. 556-566, Mar. 1991.

[4] A. Yariv, Optical Electronics in Modern Communications. New York: Oxford Univ. Press, 1997.

[5] R. Ulrich, "Representation of codirectional coupled waves," Opt. Lett., vol. 1, pp. 109-111, 1977.

[6] N. Frigo, "A generalized geometrical representation of coupled mode theory," IEEE J. Quantum Electron., vol. QE-22, pp. 2131-2140, Nov. 1986.

[7] S. K. Korotky, "Three-space representation of phase-mismatch switching in coupled two-state optical systems," IEEE J. Quantum Electron., vol. QE-22, pp. 952-958, June 1986.

[8] J. P. Gordon and H. Kogelnik, "PMD fundamentals: Polarization mode dispersion in optical fibers," in Proc. Nat. Acad. Sci., vol. 97, 2000, pp. $4541-4550$

[9] A. Syahriar, V. M. Schneider, and S. Al-Bader, "The design of mode evolution couplers," J. Lightwave Technol., vol. 16, pp. 1907-1914, Oct. 1998.

[10] R. R. A. Syms, "The digital directional coupler: Improved design," IEEE Photon. Technol. Lett., vol. 4, pp. 1135-1137, Oct. 1992.

[11] M. D. Feit and J. A. Fleck, Jr., "Light propagation in graded-index optical fibers," Appl. Opt., vol. 17, pp. 3990-3998, 1978. 